\title{
Diagnostic gene sequencing panels: from design to report-a technical standard of the American College of Medical Genetics and Genomics (ACMG)
}

\author{
Lora J. H. Bean, $\mathrm{PhD} \mathbb{1 0}^{1,2}$, Birgit Funke, $\mathrm{PhD} \mathbb{1}^{3,4}$, Colleen M. Carlston, $\mathrm{PhD} \mathbb{1}^{5}$, \\ Jennifer L. Gannon, MD ${ }^{6,7}$, Sibel Kantarci, PhD ${ }^{8}{ }^{8}$, Bryan L. Krock, PhD ${ }^{9}$, Shulin Zhang, MD, PhD ${ }^{10}$ and \\ Pinar Bayrak-Toydemir, MD, PhD ${ }^{11,12}$; on behalf of the ACMG Laboratory Quality Assurance \\ Committee
}

\begin{abstract}
Disclaimer: This technical standard is designed primarily as an educational resource for clinical laboratory geneticists to help them provide quality clinical laboratory genetic services. Adherence to this standard is voluntary and does not necessarily assure a successful medical outcome. This standard should not be considered inclusive of all proper procedures and tests or exclusive of other procedures and tests that are reasonably directed to obtaining the same results. In determining the propriety of any specific procedure or test, the clinical laboratory geneticist should apply his or her own professional judgment to the specific circumstances presented by the individual patient or specimen.

Clinical laboratory geneticists are encouraged to document in the patient's record the rationale for the use of a particular procedure or test, whether or not it is in conformance with this standard. They also are advised to take notice of the date any particular standard was adopted, and to consider other relevant medical and scientific information that becomes available after that date. It also would be prudent to consider whether intellectual property interests may restrict the performance of certain tests and other procedures.
\end{abstract}

Gene sequencing panels are a powerful diagnostic tool for many clinical presentations associated with genetic disorders. Advances in DNA sequencing technology have made gene panels more economical, flexible, and efficient. Because the genes included on gene panels vary widely between laboratories in gene content (e.g., number, reason for inclusion, evidence level for gene-disease association) and technical completeness (e.g., depth of coverage), standards that address technical and clinical aspects of gene panels are needed. This document serves as a technical standard for laboratories designing, offering, and reporting gene panel testing. Although these principles can apply to multiple indications for genetic testing, the primary focus is on diagnostic gene panels (as opposed to carrier screening or predictive testing) with emphasis on technical considerations for the specific genes being tested. This technical standard specifically addresses the impact of gene panel content on clinical sensitivity, specificity, and validity-in the context of gene evidence for contribution to and strength of evidence for gene-disease association-as well as technical considerations such as sequencing limitations, presence of pseudogenes/ gene families, mosaicism, transcript choice, detection of copynumber variants, reporting, and disclosure of assay limitations.

Genetics in Medicine (2020) 22:453-461; https://doi.org/10.1038/s41436019-0666-Z

Keywords: gene panel sequencing; technical standard; design; genetic testing; next-generation sequencing (NGS)

\section{INTRODUCTION}

Gene panels developed by clinical molecular laboratories assess multiple potential genetic causes of a suspected disorder(s) simultaneously and reduce the cost and time of diagnostic testing. Gene panels are useful to diagnose disorders with genetic and clinical heterogeneity. Panels for phenotypically related disorders can increase the likelihood of identifying an underlying genetic cause and may be preferred to exome or genome sequencing to maximize target coverage and avoid secondary findings. ${ }^{1,2}$ Due to differences in decision-making processes in the absence of clear professional standards, genes included on similar disease-focused panels vary between laboratories. With the ability to sequence multiple genes simultaneously, it is imperative to evaluate critically the validity of gene-disease associations prior to test design. While the Clinical Genome Resource (ClinGen;

\footnotetext{
${ }^{1}$ Department of Human Genetics, Emory University, Atlanta, GA, USA; ${ }^{2}$ EGL Genetics, Tucker, GA, USA; ${ }^{3}$ Department of Pathology, Harvard Medical School/Massachusetts General Hospital, Boston, MA, USA; ${ }^{4}$ Veritas Genetics, Danvers, MA, USA; ${ }^{5}$ School of Medicine, University of California, San Francisco, CA, USA; ${ }^{6}$ Division of Clinical Genetics, Children's Mercy Hospital, Kansas City, MO, USA; ${ }^{7}$ Department of Pediatrics, University of Missouri-Kansas City School of Medicine, Kansas City, MO, USA; ${ }^{8}$ Quest Diagnostics Nichols Institute, San Juan Capistrano, CA, USA; ${ }^{9}$ Division of Genomic Diagnostics, The Children's Hospital of Philadelphia, Philadelphia, PA, USA; ${ }^{10}$ Department of Pathology and Laboratory Medicine, University of Kentucky, Lexington, KY, USA; ${ }^{11}$ Department of Pathology, University of Utah, Salt Lake City, UT, USA; ${ }^{12}$ ARUP Laboratories, University of Utah, Salt Lake City, UT, USA. Correspondence: ACMG (documents@acmg.net)

Board Approval: The Board of Directors of the American College of Medical Genetics and Genomics approved this technical standard on 19 July 2019.
} 
www.clinicalgenome.org) has published a framework for establishing the validity of gene-disease relationships, ${ }^{3}$ it does not give details on how level of evidence should be used when designing a gene panel. This report provides guidance on gene selection, reviews and gives options for handling technically challenging sequence variants, and recommends auxiliary testing when sequencing methods are technically inadequate for diagnostic gene panels. The same principles apply to ad hoc gene panels (e.g., limited analysis of exome or genome sequencing data) based on the patient's clinical features, and small Sanger sequencing based panels. As gene panels increasingly serve as first-line testing for many genetic disorders, such standards are critical to harmonize testing across diagnostic laboratories, maintain a high standard of quality, and decrease the time to diagnosis for patients.

\section{METHODS}

In 2016, a workgroup of American College of Medical Genetics and Genomics (ACMG) members with clinical laboratory expertise convened to develop recommendations on gene panel design and reporting. The workgroup evaluated the literature for existing standards and technical considerations in the development of gene panels. Expert opinion included the coauthors of the document and members of the ACMG Laboratory Quality Assurance Committee. Any conflicts of interest for workgroup members or consultants are listed. A draft was delivered to the ACMG Board of Directors for review and member comment. The draft document was posted on the ACMG website and an email link was sent inviting ACMG members to provide comment. All comments were assessed by the authors. When appropriate, additional evidence was included to address member comments and the draft was amended. Both member comments and author responses were reviewed by a representative of the ACMG Laboratory Quality Assurance Committee and by the ACMG Board of Directors. The final document was approved by the ACMG Board of Directors. This report is intended to serve as a general framework that can be used for any diagnostic gene panel. Depending on the gene and the condition, additional considerations may apply to ensure maximum technical and clinical validity.

\section{Definitions}

Diagnostic gene panel: A stand-alone clinical assay intended for a specific clinical indication (i.e., features of or a family history suggestive of a genetic condition), but not carrier screening or predictive testing (i.e., testing without a clinical indication or family history), each of which has its own specific considerations.

GAD: Gene associated with Mendelian disorder; GADs include genes that meet criteria for definitive, strong, or moderate evidence for association with disease as described by ClinGen. ${ }^{3}$

GUS: Gene of uncertain significance; GUSs include genes that meet the ClinGen categories of limited or disputed evidence. $^{3}$
Table 1 Examples of phenotype-directed panels with subpanels

\begin{tabular}{ll} 
Full Panel & Subpanel \\
\hline Pan-cardio panel & Dilated cardiomyopathy \\
& Hypertrophic cardiomyopathy \\
Hearing loss & Nonsyndromic hearing loss \\
& Usher syndrome \\
Intellectual disability (ID) & Nonsyndromic ID \\
& Syndromic ID \\
& X-linked ID \\
\hline
\end{tabular}

Clinical testing: Diagnostic, predictive, carrier, or prenatal testing intended for use in medical management performed in a laboratory holding, at a minimum, a CLIA license in the United States or equivalent accreditation in other jurisdictions.

\section{PANEL DESIGN CONSIDERATIONS} Phenotype-directed diagnostic gene panels

Due to the sequencing capacity of current technologies, hundreds to thousands of genes can now be delved simultaneously to determine the cause of genetic disorders. Examples of several phenotype-directed panels and subpanels are listed in Table 1. The goal of a diagnostic gene panel is to maximize clinical sensitivity and minimize the clinical burden from analysis of inappropriate or unnecessary genes that may result in variants of uncertain clinical significance (VUS). Patients may have limited opportunity for serial genetic tests, arguing for casting a wide diagnostic net; however, long lists of (VUS), a possible outcome from testing large numbers of genes, can complicate medical management and cause unnecessary patient anxiety. When a panel is well-designed, it will

- Be cost-effective for a particular clinical indication.

- Maximize clinical sensitivity by, to the extent possible, including all GADs associated with a disorder, thereby allowing disorders with clinical heterogeneity and overlapping features to be molecularly diagnosed.

- Include GUSs with limited but emerging evidence if additional criteria are met (see example in "Clinical sensitivity," "Gene considerations," and "Reporting").

- Maximize clinical specificity by limiting or excluding GUSs, thereby minimizing detection of VUS.

- Employ auxiliary assays for genes/regions that cannot be interrogated with current sequencing technology to maximize the clinical utility.

\section{Clinical sensitivity}

For a gene panel to have a high clinical sensitivity, the indication for testing must have a genetic cause among a reasonably high proportion of cases. The clinical sensitivity of gene panels is primarily limited by the incompleteness of biomedical knowledge of relevant gene-disease associations, as well as a laboratory's technical ability to detect all disease-causing variants. Diagnostic gene panels should include all GADs. 
Although broad inclusion of GUSs in diagnostic panels is discouraged, there are scenarios where inclusion may be meaningful. For example, a gene that has been recently reported in association with disease may be useful as additional evidence may emerge quickly. If a panel includes GUSs with limited but emerging evidence, some additional requirements for reporting must be met (see "Gene considerations" and "Reporting"). Panel content should be monitored and revised when new disease genes that meet criteria for inclusion are discovered. This can include, but is not limited to, consultation with disease experts, regular literature surveys, or review of gene-specific resources. Ideally, the laboratory bioinformatics pipeline could be configured to flag and present new database (e.g., OMIM, PubMed, Human Genome Mutation Database [HGMD]) entries; alternatively a manual review cycle of 6 months is recommended.

The spectrum of pathogenic variant types should be considered for any gene to maximize assay sensitivity. The reported frequency of single-nucleotide variants (SNVs), intraexonic insertions and deletions, intragenic (multiexonic) copy-number variants (CNVs), repeat expansions, or structural aberrations ought to be prioritized. When sequencing is not an appropriate testing methodology to detect variants with an impact on clinical sensitivity (Table $2 \mathbf{a}$ ), the use of alternative testing is needed at some point in the testing process. If a non-sequencing-based genetic test is part of a standard initial clinical workup, diagnostic panels may offer flexible ordering options, such as with or without auxiliary assays, to reduce costs and testing redundancy. If auxiliary assays are not part of the panel option, limitations of the panel should be disclosed, and testing recommendations for auxiliary assays made in both the test description and the clinical report.

\section{Clinical specificity}

While it may be technically possible to sequence all genes related to a phenotype, the power of a gene panel is the ability to match a patient's specific clinical features to genes associated with that phenotype, thereby increasing clinical specificity and limiting the number of VUS. Disorders with extreme genetic heterogeneity may become unwieldy as a single comprehensive test. For such disorders, exome or genome sequencing may be a better initial test with the option to reflex to larger multidisease panels or exome or genome sequencing if first-line testing is nondiagnostic (Table $\mathbf{2 b}$ ). The opposite extreme is also worth noting, and allele-specific testing may be the most efficient approach in certain scenarios. Although testing has moved away from ethnicity-based testing recommendations, targeted testing has better specificity for individuals of traditionally well-studied ethnic groups, such as the Amish and Mennonite populations. ${ }^{4}$

\section{Clinical validity}

While the genetics community has long recognized the need for providing frameworks to establish the clinical interpretation of sequence variants, ${ }^{5}$ extending this mindset to gene-disease relationships has only recently gained traction. The Clinical Genome Resource (ClinGen) is driving efforts to provide standards for establishing the clinical validity of gene-disease associations with a framework that ranks the evidence from "definitive" to "refuted," and this is being incorporated into practice by the genetics community. ${ }^{3,6-8}$ Understanding the validity of a gene-disease association is the first step in selecting appropriate genes to be tested in a clinical setting. Clinical utility of gene sequencing decreases as the evidence for a disease association decreases. Laboratories are responsible for evaluating how the strength of evidence for gene-disease correlation may limit their ability to classify variants as pathogenic or likely pathogenic. For example, variants predicted to be loss-of-function in moderate evidence genes will rarely exceed a classification above likely pathogenic given that the association to disease is still building, and if genes of limited but emerging evidence are included, variants should not be classified above VUS, even if a variant is predicted to be loss-of-function. The working group recommends laboratories utilize the ClinGen gene evidence framework $^{3}$ and the American College of Medical Genetics and Genomics and Association for Molecular Pathology (ACMGAMP) standards for variant interpretation, ${ }^{5}$ as well as regularly conduct systematic review of genes for emerging evidence. For a diagnostic gene panel, GADs with evidence for the clinical indication of the test would be included (see Table 3 ). Subsequent considerations are driven by the intended use of the test (e.g., diagnostic, predictive, or carrier screening).

Special requirements should be met if a gene panel that includes GUSs for exploratory (e.g., limited analysis of exome data referred to as a panel) or research purposes. Since GUSs have little or no evidence for clinical validity, a test that includes GUSs should make this clear in the test description (see "Test description/test name").

\section{Gene contribution}

\section{GENE CONSIDERATIONS}

For most genetic disorders, overall contribution to disease is distributed unevenly among different genes. Often pathogenic variants in a small number of genes contribute to the majority of cases while many genes represent rarer genetic etiologies. For example, in hypertrophic cardiomyopathy, pathogenic and likely pathogenic variants in the MYBPC3 and MYH7 genes account for approximately $80 \%$ of molecular diagnoses, while panels may include over 50 genes in an attempt to diagnose rare causes of $\mathrm{HCM}^{9}$ Nonsyndromic hearing loss, ${ }^{10}$ retinitis pigmentosa, ${ }^{11}$ and Stickler syndrome ${ }^{12}$ are other examples with similar genetic distributions. Whenever technically feasible, laboratories should include an option for testing all GADs, though this may be offered as a reflex option following a smaller, more specific panel as mentioned previously (see "Clinical specificity"). Genes most commonly responsible for disease could be in a smaller panel, and genes with a more infrequent contribution to disease then included in a reflex panel. Accurate estimates of disease contribution may not be possible for newly discovered genes, such as included GUSs with limited but emerging evidence (see "Clinical sensitivity"), or rare genetic causes of a condition 


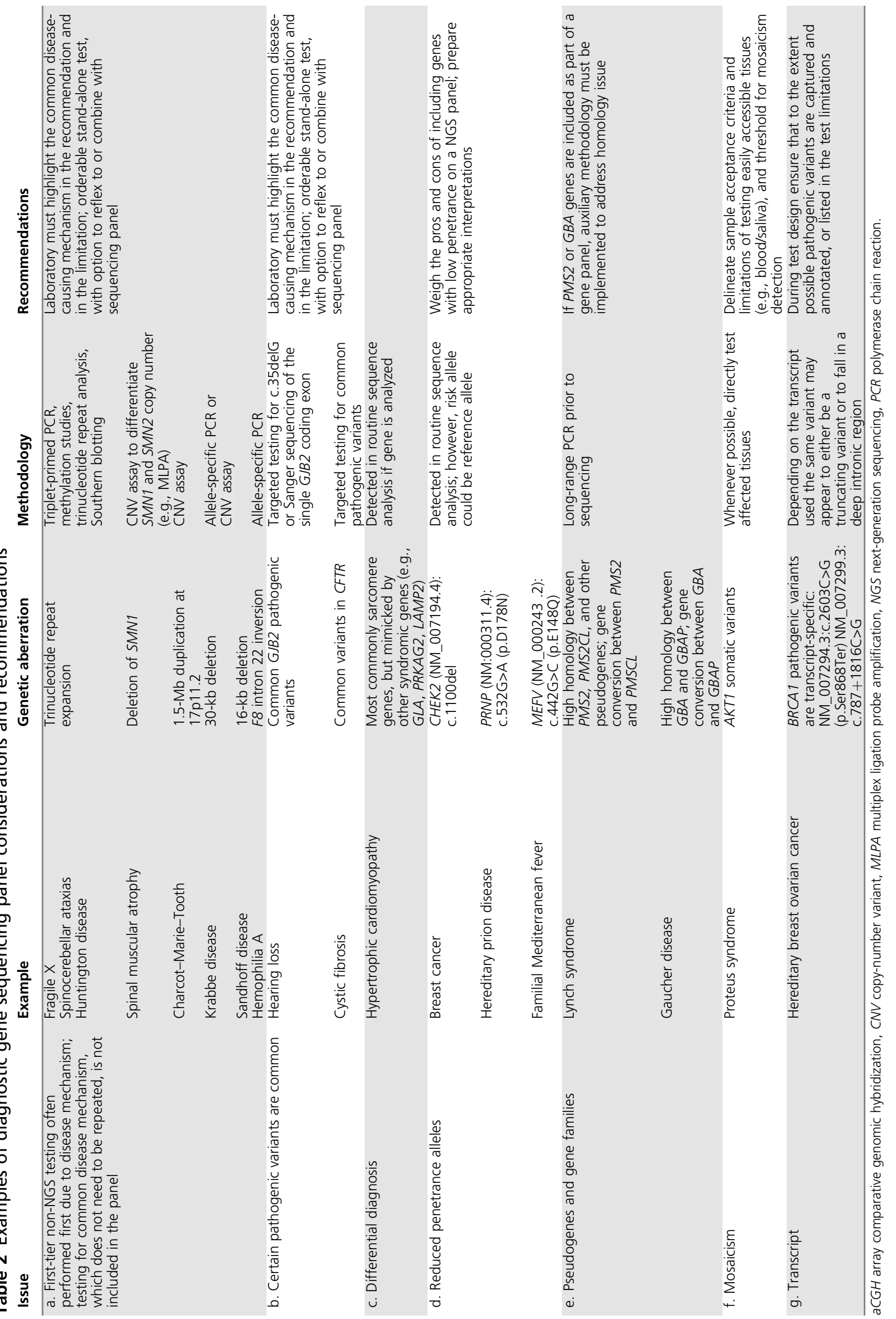


Table 3 Inclusion criteria for genes with various gene-disease evidence levels comparing diagnostic gene panels with exome/genome testing

\begin{tabular}{|c|c|c|c|c|c|c|c|}
\hline \multirow{2}{*}{$\begin{array}{l}\text { ClinGen framework }^{3, a} \\
\text { Gene category }\end{array}$} & \multirow[t]{2}{*}{ 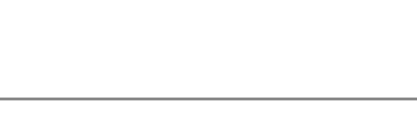 } & \multirow{2}{*}{$\begin{array}{l}\text { Definitive } \\
\text { Genes assc }\end{array}$} & \multirow{2}{*}{\multicolumn{2}{|c|}{$\begin{array}{l}\text { Strong Moderate } \\
\text { ciated with disease }\end{array}$}} & \multicolumn{2}{|l|}{ Limited } & \multirow{2}{*}{$\begin{array}{l}\text { No } \\
\text { evidence }\end{array}$} \\
\hline & & & & & \multicolumn{2}{|c|}{ Genes of uncertain significance } & \\
\hline Test purpose & $\begin{array}{l}\text { Predominant diagnostic } \\
\text { approach }\end{array}$ & & & & $\begin{array}{l}\text { Genes with } \\
\text { emerging } \\
\text { evidence }\end{array}$ & $\begin{array}{l}\text { Genes with no } \\
\text { emerging } \\
\text { evidence }\end{array}$ & $\begin{array}{l}\text { Genes } \\
\text { with no } \\
\text { evidence }\end{array}$ \\
\hline $\begin{array}{l}\text { Confirmation of } \\
\text { clinical Dx }\end{array}$ & $\begin{array}{l}\text { Disease-focused multigene panel; } \\
\text { other non-sequencing-based } \\
\text { ancillary assays }\end{array}$ & Include & Include & Include ${ }^{b}$ & $\begin{array}{l}\text { Typically } \\
\text { exclude }\end{array}$ & Exclude & Exclude \\
\hline $\begin{array}{l}\text { Establish genetic } \\
\text { diagnosis for clinically } \\
\text { complex cases }\end{array}$ & Exome/genome $^{e}$ & Include & Include & Include ${ }^{b}$ & \multicolumn{3}{|c|}{ Additional requirements $^{d}$} \\
\hline \multicolumn{8}{|c|}{$\begin{array}{l}\text { Genes with conflicting evidence reported (disputed, refuted) are not appropriate for diagnostic gene panels. } \\
\text { Indicate in the report that evidence for the disease association is still building. Variants are unlikely to be classified above likely pathogenic. } \\
\text { Although broad inclusion of genes of uncertain significance (GUSs) in diagnostic panels is discouraged, there are scenarios where inclusion may be meaningful (see } \\
\text { liscussion in "Clinical sensitivity"). } \\
\text { Report with specific statement that disease association and inheritance has not been established. Results from these genes should be separated from the clinical result } \\
\text { o the extent possible within the reporting system. } \\
\text { Consent process specific to exome/genome testing required. }\end{array}$} \\
\hline
\end{tabular}

such as GADs with only moderate evidence. The laboratory must evaluate the evidence available and decide, based on the intended scope or gene panel description, which genes will be included on the panel.

\section{Differential diagnosis}

For disorders with significant clinical overlap, one strategy to maximize clinical sensitivity is to design a panel that includes genes recognized to be causative of disorders in the differential diagnosis of a phenotype (Table 2c). It is important to note that genetic syndromes may present with an isolated finding, either due to young age when additional features have yet to be manifested or by chance (i.e., variable expressivity). Therefore, genes associated with syndromes should be considered for inclusion in gene panels for the characteristic phenotype. For example, familial hypertrophic cardiomyopathy (HCM) is typically caused by heterozygous pathogenic variants in sarcomere genes. However, syndromic disorders such as Fabry disease (GLA pathogenic variants), lethal congenital glycogen storage disease of the heart (PRKAG2 pathogenic variants), and Danon disease (LAMP2 pathogenic variants) may mimic familial HCM. ${ }^{13}$ Furthermore, RASopathies such as Costello and cardiofaciocutaneous syndromes are caused by pathogenic variants in RAS/MAPK pathway genes. RASopathies are associated with variable degrees of intellectual disability, developmental delay, dysmorphic features, ectodermal abnormalities, increased risk for certain malignancies, and congenital heart disease, particularly HCM and pulmonic stenosis. ${ }^{14,15}$ When genes are associated with syndromes that have substantial clinical overlap with isolated presentations, such as those syndromes that include HCM or hearing loss, they are often included in clinical panels, a practice that is recognized as effective for establishing molecular diagnoses.
Genes included as part of the differential diagnosis should be GADs for the related disorder, but may have limited or even no evidence for the test-specific clinical indication. If a pathogenic variant were detected in such a gene, the expectation would be that a diagnosis of the disorder primarily associated with that gene would be considered.

\section{Incomplete penetrance and disease association}

In addition to genes and variants with complete or high penetrance, some phenotypes or syndromes are caused by genes or variants with low penetrance (Table 2d). While it is technically feasible to include genes with low-penetrance pathogenic variants on gene panels, the penetrance and the factors affecting penetrance are generally not known, thus limiting clinical utility. Family history alone is generally not sufficient to predict penetrance. For example, variable penetrance within the same family has been described for breast cancer, hereditary prion disease, and familial Mediterranean fever. ${ }^{16-18}$ It is prudent to consider that while including genes with low-penetrance variants on a gene panel may increase diagnostic sensitivity, if clinical features are not due to the lowpenetrance allele, an incorrect diagnosis may be reached and treatment initiated; however, if a gene that contributes significantly to overall disease (e.g., familial Mediterranean fever caused by $M E F V$ variants among periodic fever syndromes) is excluded due to incomplete penetrance, a diagnosis may be missed. Contribution to disease by such genes must be weighed against the risk that such testing may create unnecessary anxiety among patients and family members. Evidence for incomplete penetrance of variants must be clearly explained in the clinical report; see "Gene information."

Genes with non-Mendelian disease association present an additional challenge. One example would be disclosing Crohn disease-associated variants in NOD2 on a panel for Mendelian 
causes of bowel disease. As more data on the clinical utility of variant analysis in polygenic traits and the calculation of polygenic risk scores become available, incorporating this information into a diagnostic panel may become feasible; however, these data must be clearly delineated as separate from genes and variants associated with Mendelian disorders.

\section{Exclusion criteria}

GUSs are typically not included on a panel. However, if GUSs are included, the data must be treated differently from GADs. The test description, consenting process, disclaimers, and information reported should make clear that one or more genes with unproven disease association are being included.

If genes selected through pathway analysis ("candidate genes") or other predictions without further proof of valid disease association are included, the following criteria should be applied:

- Patients' consent for testing should be similar to exome/ genome sequencing or research to properly disclose the possibility of inconclusive results.

- Variants reported in GUSs should be separated from those reported in GADs to the extent allowed by the reporting system. If separate sections are not possible, data from GUSs should be moved to a separate paragraph/text section.

Variants reported in GUSs should not be associated with specific genetic disorders or inheritance patterns (see "Reporting"); rather, a brief summary of gene data may be given (e.g., "A single missense variant in this gene has been shown to segregate with [phenotype] in one family; however, there is insufficient evidence to determine the clinical significance of variants in this gene.").

\section{TECHNICAL CONSIDERATIONS}

Once a clinically appropriate gene panel is defined, technical considerations must be explored. It is important to recognize gene characteristics that may complicate testing or interpretation and when other technologies may be needed to cover adequately the spectrum of pathogenic variants of a particular disorder. Additionally, the performance of the sequencing methodology (e.g., amplification versus capture prior to NGS, exome versus genome sequencing) may vary for genes of specific interest; therefore, gene sequencing performance must be evaluated and validated by the testing laboratory.

\section{Sequence limitations}

Some genomic regions are challenging to sequence. In addition to highly homologous regions, regions of high and low GC content (high GC content is often present in the 5' region including the first exon), homopolymers, and repeats of any size cannot be sequenced with high confidence or quality. This can lead to inadequate coverage by NGS; therefore, special attention needs to be placed on the relative importance of such regions (assessed by gene evidence). The following approaches should be used to manage low-coverage areas:
- Mutational hotspots and sites of common founder variants, if not well covered, should be filled in by orthogonal technologies to ensure adequate clinical sensitivity.

- If the laboratory's policy includes fill-in sequencing for insufficiently covered regions, regions that are not critical for optimal clinical sensitivity (e.g., regions within minor contribution to disease or genes added to the panel as part of a broader differential diagnosis) may be removed from the test and such limitations disclosed in the test description and on the report.

- Regions not covered in a specific case must be disclosed either in summary (e.g., percent of bases not covered) or in detail (e.g., exon 1 of gene EHMT1); details must be available upon request.

CNV detection by NGS technologies may be included as part of the panel (see "Copy-number variants"); however, if not included, the proportion of pathogenic variants expected to be CNVs not detected by the analysis should be stated as a limitation.

\section{Pseudogenes and gene families}

Current NGS technology does not allow interrogation of nonunique sequences such as duplicated (e.g., repeated exons such as WRN [NM_000553.4] exons 10 and 11) or repetitive sequences (e.g., dinucleotide, trinucleotide repeats). Medically relevant, nonunique sequences include genes that have coding sequence repeats such as processed or duplicated pseudogenes. Processed pseudogenes are less likely to interfere with read alignment since exons in the original gene can often be anchored by unique intronic regions; however, duplicated pseudogenes, which have homology across exonic and intronic regions, more commonly interfere with read alignment. If a gene with a pseudogene accounts for a significant proportion of cases, auxiliary methodologies must be employed to ensure clinical sensitivity (Table 2e). These may include long-range polymerase chain reaction (PCR) followed by sequencing (Sanger or NGS) or emerging technologies that are designed to circumvent these issues. Special effort should be made during test design and validation to identify highly homologous regions. ${ }^{19,20}$ Such regions should be excluded from the interrogated region unless the NGS methodology is thoroughly validated or variant calls are confirmed by orthogonal methods.

\section{Mosaicism}

For genes in which somatic mosaicism is the most common disease mechanism (Table $\mathbf{2 f}$ ), the preferred sample type (e.g., affected versus unaffected tissue, blood, etc.) should be stated in the sample acceptance criteria. Testing limitations of easily accessible tissue should be explained (e.g., blood or saliva). If a test is specifically designed to detect mosaicism, the laboratory must establish the minimum allele fraction threshold of detection given the average coverage of the panel, and provide this information in the report (see "Gene information"). 


\section{Transcripts}

Many genes have multiple transcripts that include or exclude different exons, and based on which transcript is used for reporting, the variant call and its predicted effect on the gene/ protein can vary dramatically (Table $\mathbf{2 g}$ ). Although efforts are being made by the National Center for Biotechnology Information (NCBI) and Ensembl/GENCODE to create consensus transcripts through the Matched Annotation by NCBI and EMBL-EBI (MANE) project, laboratories must currently make their own determination of which transcripts will be interrogated. ${ }^{21}$ A College of American Pathologists (CAP) and Association for Molecular Pathology (AMP) working group recently provided a practical guide for carrying out transcript evaluation. ${ }^{8}$

When a gene is well-characterized, as determined by a laboratory based on the peer-reviewed literature, professional focus groups, and/ or experts in the field:

- Laboratories should adhere to the conventions within the field for transcript isoforms, which favor transcripts used in many publications and/or known to have biological relevance based on the peer-reviewed literature and locusspecific databases.

- Determine if significant discrepancies exist in published/ posted transcripts or naming conventions to ensure that bioinformatics processes are designed to handle this information.

When a gene is not well-characterized:

- Transcript accession numbers and versions used in publications should be included to ensure that all reported pathogenic variants would be detected.

- When information about a gene is limited, an all-exon approach across one or more transcripts, with the largest canonical transcript as the reporting default, should be used.

Exons in rarely expressed transcripts, present in a minority of transcripts, or solely predicted by in silico algorithms should be included for analysis only with caution and variants in such exons must be interpreted with caution; however, assay design should include reported transcripts as completely as possible since assay design may be difficult to change and more information may become available with time, making inclusion of previously excluded exons desirable.

More than one transcript may need to be used if there is no single, long transcript to cover all exons with variants identified in affected individuals. However, alternate transcripts may have disease-specific consequences. A list of transcripts analyzed, including primary and alternate transcripts, should be provided (see "Gene information"). Of note, some reported pathogenic or likely pathogenic variants are located deep in intronic ( $> \pm 20$ base pairs from the exon) regions or in untranslated regions. Those regions, as possible, should be targeted for assay inclusion.

\section{Copy-number variants}

CNV analysis using NGS data is still improving with available algorithms variable in their sensitivity and specificity. The false positive rate can be high and is generally proportional to the depth and uniformity of coverage and inversely proportional to the size of the CNV and the sequence itself, especially for small, intragenic exon-level deletions and duplications. ${ }^{22-25}$ Genome data has a lower false positive and false negative $\mathrm{CNV}$ detection rate compared with capture of amplification-based enrichment methods; however, gene panel analysis as a stand-alone test from genome sequencing is likely to remain cost-prohibitive for some time. Therefore, careful validation experiments must be conducted to establish the size of CNV below which accuracy is compromised and confirmatory testing by orthogonal methods is necessary. Reports must clearly state limitations surrounding CNV detection and specify what CNV size (expressed as the number of consecutive exons) can be reliably detected (see "Limitations").

\section{REPORTING}

General standards for reporting of NGS data have been put forth by the ACMG. ${ }^{26}$ Because the vast majority of gene panels are performed using NGS technology, these standards would apply with a few special considerations.

\section{Test description/test name}

The laboratory is responsible for providing a summary of the intended use of a gene panel (the clinical indications), expected clinical sensitivity and specificity (if known), and a list of genes included on the panel. Test information should clearly indicate:

- Appropriate clinical indications.

- Scope of gene panel: is the test a narrower subpanel or broader comprehensive panel (see Table 1 for examples); are genes associated with both syndromic and nonsyndromic conditions included.

- Inclusion, if any, of GUSs that make the panel an exploratory panel or a panel that has specific consent requirements.

\section{Gene information}

Genes should be referred to using official gene names (common aliases may be included if historically well known). For gene with a reported variant, the report must include:

- Transcript analyzed: Disclosure of all transcripts analyzed on the report is preferred. However, for large panels $(\sim 100$ genes or as allowed by the reporting system), transcripts may be provided on the laboratory website. Note: since transcripts analyzed change over time, provisions must be made that allow a report to be matched to transcripts that were used at the time the test was performed.

- Gene-disease association.

- Inheritance.

- Incomplete penetrance (if applicable). 
Detailed gene-level information on disease association, inheritance, incomplete penetrance (if applicable), and level of gene evidence may be provided via the laboratory website or upon request.

\section{Limitations}

Transparency is imperative when performing a gene sequencing panel so that ordering providers know what the test includes and what it does not. At a minimum, any low-coverage or nontargeted exonic regions (see "Sequence limitations") must be listed on the report or a summary provided with specific instructions on how to obtain detailed information. When reporting results from a gene panel, the methodologies used and test limitations must be clearly delineated. It is the responsibility of the laboratory to identify regions/exons that perform poorly or inconsistently during test validation in a disclaimer or limitations section either in summary or in detail (see "Mosaicism"). In addition, if regions or genes were specifically excluded from testing (e.g., because they are recommended as first-tier tests or due to the presence of pseudogenes), this should be specified. Technical limitations for detection of large deletions/duplications and noncoding variants should be explained in detail.

\section{SUMMARY}

Diagnostic gene panels require careful thought around intended use, design, and implementation. This document provides a workflow for ensuring clinical sensitivity, specificity, and validity through systematic review of genetic causes of disease and strength of gene-disease evidence (Box 1 and Fig. 1). Laboratories are ultimately responsible for the design, validation, data generation, interpretation, and reporting of gene panels as clinical assays. The laboratory must consider

\section{Box 1}

Phenotype-directed panel testing

- Ensure clinical validity and sensitivity.

- Maximize clinical specificity.

- Employ auxiliary assays as appropriate.

- Include genes with sufficiently established disease associations.

\section{Gene considerations}

- Diagnostic gene panels should include all GADs.

- GADs associated with disorders in the differential diagnosis may be included on a diagnostic gene panel.

- GUSs and genes/variants associated with variable penetrance require special consent and/or reporting procedures.

Technical consideration

- Ancillary methods should be employed as appropriate to interrogate. - GADs with a pseudogene that are a disease contributor.

- Repeat expansions not detectable by sequencing.

- Common deletions or duplications not detected by the NGS assay.

- Mutational hotspots and common founder variants must be well covered or filled in by Sanger sequencing.

- Transcripts, selected based on knowledge about the gene, should be listed on the report.

Reporting

- The intended use (clinical indication) of the test is stated.

- Detailed gene-level information may be provided via the laboratory website or upon request.

- Methodologies included and test limitations must be clearly stated.

- Regions of genes not sequenced (excluded) or missing coverage should be disclosed.

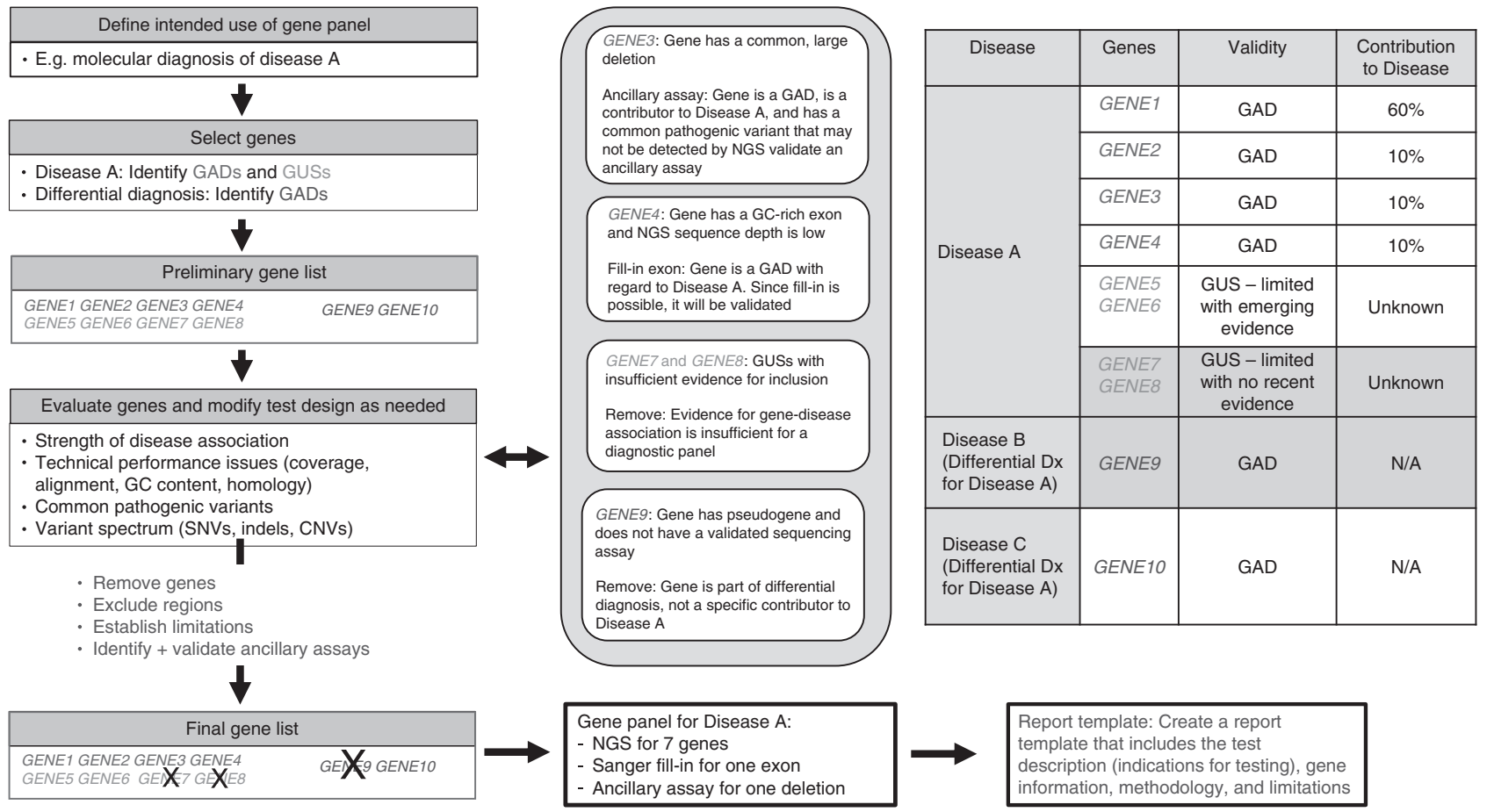

Fig. 1 Workflow for design, evaluation, and implementation of a diagnostic gene panel. CNV copy-number variant, GAD gene associated with Mendelian disorder, GUS gene of uncertain significance, SNV single-nucleotide variant. 
effectiveness of NGS assays and either augment NGS testing through the use of ancillary assays or disclose limitations of the methodology given the design of the gene panel. By looking beyond the bulk of NGS data to the higher clinical sensitivity achieved by ensuring completeness of testing based on strength of gene-disease evidence, the laboratory can design and implement a complete high-quality diagnostic gene panel test.

\section{DISCLOSURE}

L.J.H.B., B.F., S.K., B.L.K., S.Z., and P.B.T. are directors of clinical laboratories that offer molecular genetic testing panels on a feefor-service basis. C.M.C. and J.L.G. declare no conflicts of interest.

Publisher's note Springer Nature remains neutral with regard to jurisdictional claims in published maps and institutional affiliations.

\section{REFERENCES}

1. Klein CJ, Foroud TM. Neurology individualized medicine: when to use next-generation sequencing panels. Mayo Clin Proc. 2017;92:292-305.

2. Bevilacqua J, Hesse A, Cormier B, et al. Clinical utility of a 377 gene custom next-generation sequencing epilepsy panel. J Genet. 2017; 96:681-685.

3. Strande NT, Riggs ER, Buchanan AH, et al. Evaluating the clinical validity of gene-disease associations: an evidence-based framework developed by the Clinical Genome Resource. Am J Hum Genet. 2017;100:895-906.

4. Strauss KA, Puffenberger EG. Genetics, medicine, and the Plain people. Annu Rev Genomics Hum Genet. 2009;10:513-536.

5. Richards S, Aziz N, Bale $S$, et al. Standards and guidelines for the interpretation of sequence variants: a joint consensus recommendation of the American College of Medical Genetics and Genomics and the Association for Molecular Pathology. Genet Med. 2015;17:405-424

6. Lu JT, Ferber M, Hagenkord J, et al. Evaluation for genetic disorders in the absence of a clinical indication for testing: elective genomic testing. J Mol Diagn. 2019;21:3-12.

7. Smith ED, Radtke K, Rossi M, et al. Classification of genes: standardized clinical validity assessment of gene-disease associations aids diagnostic exome analysis and reclassifications. Hum Mutat. 2017;38:600-608.

8. Santani A, Murrell J, Funke B, et al. Development and validation of targeted next-generation sequencing panels for detection of germline variants in inherited diseases. Arch Pathol Lab Med. 2017;141:787-797.

9. Alfares $A A$, Kelly MA, McDermott $G$, et al. Results of clinical genetic testing of 2,912 probands with hypertrophic cardiomyopathy: expanded panels offer limited additional sensitivity. Genet Med. 2015;17:880-888.

10. Shearer $A E$, Hildebrand MS, Smith RJH. Hereditary hearing loss and deafness overview. In: Adam MP, Ardinger HH, Pagon RA, Wallace SE,
Bean LH, Stephens K, Amemiya A, (eds.) GeneReviews. Seattle, WA: University of Washington; 1993-2019.

11. Fahim AT, Daiger SP, Weleber RG. Nonsyndromic retinitis pigmentosa overview. In: Adam MP, Ardinger HH, Pagon RA, Wallace SE, Bean LH, Stephens K, Amemiya A (eds.) GeneReviews. Seattle, WA: University of Washington; 1993-2019.

12. Robin NH, Moran RT, Ala-Kokko L. Stickler syndrome. In: Adam MP, Ardinger HH, Pagon RA, Wallace SE, Bean LH, Stephens K, Amemiya A (eds.) GeneReviews. Seattle, WA: University of Washington; 1993-2019.

13. Cirino AL, Ho C. Hypertrophic cardiomyopathy overview. In: Adam MP, Ardinger HH, Pagon RA, Wallace SE, Bean LJH, Stephens K, Amemiya A (eds.) GeneReviews. Seattle, WA: University of Washington; 1993-2019.

14. Jhang WK, Choi JH, Lee BH, Kim GH, Yoo HW. Cardiac manifestations and associations with gene mutations in patients diagnosed with RASopathies. Pediatr Cardiol. 2016;37:1539-1547.

15. Cao H, Alrejaye N, Klein OD, Goodwin AF, Oberoi S. A review of craniofacial and dental findings of the RASopathies. Orthod Craniofac Res. 2017;20(Suppl 1):32-38.

16. CHEK2 Breast Cancer Case-Control Consortium. CHEK2*1100delC and susceptibility to breast cancer: a collaborative analysis involving 10,860 breast cancer cases and 9,065 controls from 10 studies. Am J Hum Genet. 2004;74:1175-1182.

17. Shohat M. Familial Mediterranean fever. In: Adam MP, Ardinger $\mathrm{HH}_{\text {, }}$ Pagon RA, Wallace SE, Bean LJH, Stephens K (eds.) Gene Reviews. Seattle, WA: University of Washington; 1993-2019.

18. Synofzik MP, Bauer P, Schols L. Prion mutation D178N with highly variable disease onset and phenotype. J Neurol Neurosurg Psychiatry. 2009;80:345-346.

19. Sun $Y$, Ruivenkamp CA, Hoffer MJ, et al. Next-generation diagnostics: gene panel, exome, or whole genome? Hum Mutat. 2015;36:648-655.

20. Mandelker D, Schmidt RJ, Ankala A, et al. Navigating highly homologous genes in a molecular diagnostic setting: a resource for clinical nextgeneration sequencing. Genet Med. 2016;18:1282-1289.

21. Pujar S, O'Leary NA, Farrell CM, et al. Consensus coding sequence (CCDS) database: a standardized set of human and mouse proteincoding regions supported by expert curation. Nucleic Acids Res. 2018;46: D221-D228.

22. Ellingford JM, Campbell $C$, Barton $S$, et al. Validation of copy number variation analysis for next-generation sequencing diagnostics. Eur J Hum Genet. 2017;25:719-724.

23. Kerkhof J, Schenkel LC, Reilly J, et al. Clinical validation of copy number variant detection from targeted next-generation sequencing panels. $J$ Mol Diagn. 2017;19:905-920.

24. Yao R, Yu T, Qing Y, Wang J, Shen Y. Evaluation of copy number variant detection from panel-based next-generation sequencing data. Mol Genet Genomic Med. 2019;7:e00513.

25. Retterer K, Scuffins J, Schmidt D, Lewis R, et al. Assessing copy number from exome sequencing and exome array CGH based on CNV spectrum in a large clinical cohort. Genet Med. 2015;17:623-629.

26. Rehm HL, Bale SJ, Bayrak-Toydemir P, et al. ACMG clinical laboratory standards for next-generation sequencing. Genet Med. 2013; 15:733-747. 